polymerase.

An interesting contrast in initiation is provided by $\mathrm{G} 4$ which although closely related to $\varphi \times 174$ requires no pre-priming. One possible explanation for this difference comes from studies of the DNA sequences at the origin of replication of the complementary strand. Whereas $\varphi \mathrm{X} 174$ can initiate complementary strand synthesis at several sites, there is only one initiation site on G4. The primase alone recognises a DNA sequence coresponding to the in vivo origin of replication. This site consists of a 130-nucleotide stretch (N. Godson, Yale University) which is strongly conserved in the related phage ST-1 (which also needs no pre-priming) (D. Dressler, Harvard University) but not in $\varphi$ X174. Dressler suggested that this relatively large sequence provides the nucleic acid counterpart of the dnaB protein. G4 starts $\mathrm{RF} \rightarrow \mathrm{ss}$ replication from a completely different origin which shows sequence similarity with that of $\varphi \mathrm{X} 174$ $\mathrm{RF} \rightarrow \mathrm{ss}$ origin in gene $A$. Synthesis is initiated here by nicking one of the strands and using the base-paired $3^{\prime} \mathrm{OH}$ end generated as a primer.

Evidence for discontinuous replication in bacteria was provided in 1964 by the discovery of 'Okazaki pieces'small nascent DNA fragments of a few thousand nucleotides. However, rerecently Lehman and colleagues (see Olivera et al. Fedn Proc. 36, 654; 1977) discovered that a part at least of the pool of small DNA fragments believed to be Okazaki pieces in bacteria can result from the misincorporation and subsequent excision of uracil into DNA. Subsequently a similar possible source of fragments has been found in cultured lymphocytes (M. Goulian, Rockefeller University) and polyoma virus replication (P. Reichard, Karolinska Institute, Stockholm). However, Okazaki pieces are too attractive a solution to the problem of directionality of polymerases to be lightly discarded, and were still an article of belief for most of the participants. In theory, true Okazaki pieces should be distinguishable by their RNA-primed ends but as B. M. Olivera (Stanford University) has found, some of the common criteria for identifying RNA primers do not distinguish between authentic RNA-primed fragments and pieces generated by uracil excision. Until it is possible to measure the contribution of RNA-primed fragments to the pool the extent of postreplication excision and repair remains uncertain.

However, replication of the doublestranded phages $\mathrm{T} 4$ and $\mathrm{T} 7$ provides a source of RNA-primed fragments for primer analysis. T. Okazaki (Nagoya University) described the analysis of the RNA primers on $\mathrm{T} 7$ 'Okazaki

\section{New transposons}

from J. R. Saunders

It has been known for some years that genes specifying antibiotic resistance in bacteria are often found as components of translocatable genetic elements called transposons (see Cohen Nature 263, 731; 1977). Recent work from several laboratories now suggests that functions other than drug and heavy metal ion resistance may be encoded on transposons. Recently it was reported (Nature, Jacoby et al. 274, 179; 1978) that genes for the breakdown of xylenes and toluenes carried on TOL plasmids of Pseudomonads (see News and Views 269, 470; 1977) also act as if they were part of a transposon. The tol genes are apparently borne on a translocatable element which is much larger at about $38 \mathrm{Mdal}$ than any known drug resistance transposon. It will therefore be of some interest to determine the molecular structure of this transposon with particular reference to possible flanking repeat sequences. The ability of tol function to be transposed between replicons would explain both the heterogeneity observed amongst TOL plasmids and the deletion of tol genes which sometimes occurs when $\mathrm{TOL}^{+}$strains of Pseudomonas putida are grown on benzoate (see News and Views 269, 470; 1977).

The existence of a further transposon has been demonstrated by studying $\mathrm{Lac}^{+}$plasmids. Such plasmids are of some importance clinically since they confer on host bacteria the ability to ferment lactose. This obscures a character of great significance in the routine identification of enteric pathogens such as Salmonellae which are normally lactose non-fermenting. A $\mathrm{Lac}^{+}$plasmid isolated from Yersinia enterocolitica has been shown recently to contain a transposon carrying the lac $i, z$ and $y$ genes (Cornelis et al., Mol. gen. Genet. 160, 215; 1978). This lac operon is homologous with the lactose operon of Escherichia coli except that it probably lacks the lac a (galactoside transacetylase) gene. Unlike the lac operon of E. coli which is not apparently transposable, the lactose operon on this $\mathrm{Lac}^{+}$plasmid is flanked by short (about 0.15 kilobase)

J. R. Saunders is a lecturer in the Department of Microbiology at the University of Liverpool.

pieces' formed in vivo. She finds that the majority of the RNA consists of AC-rich oligonucleotides ranging from dimers to pentaucleotides. The in vitro synthesised $\mathrm{T} 7$ primers found by inverted repeat sequences as might be expected of a transposon. These findings raise some fundamental questions as to the origin of the classical lactose operon. For instance, did the lac operon originate in E. coli and subsequently become incorporated into a transposon to assist its dissemination on plasmids to other enterobacteria? Alternatively, was the lac transposon acquired from some other organism early in the evolution of $E$. coli? Subsequent loss of transposability must then have been necessary to stabilise the $\mathrm{Lac}^{+}$character. The answer to these questions may well become clearer when many more $\mathrm{Lac}^{+}$plasmids have been investigated.

Plasmids specifying bacterial toxins are obviously important in determining the spread of pathogenic traits amongst the bacterial population. Of particular interest currently are enterotoxin (Ent) plasmids which confer on $E$. coli the ability to cause diarrhoeal disease. At the recent EMBO workshop on Plasmids in Berlin, Magdalene So (University of California, San Francisco) presented evidence which indicates that the gene determining the heat stable (ST) enterotoxin of E. coli is part of a transposon. This gene is flanked by inverted repeats of IS $l$. However, showing transposition per se is not easy because there is no readily selectable genetic marker for enterotoxin production. This problem has now been overcome by artificially splicing a resistance marker into the middle of the ST gene carried by an Ent plasmid. A non-transposable tetracycline-resistance gene was inserted into an EcoRI restriction site in the ST gene. The subsequent transposition of tetracycline resistance from this engineered plasmid must therefore be due to the transposition of the toxin gene. This technique could obviously be applied more widely to show whether or not other bacterial genes lacking an easily recognisable phenotype are borne on transposons.

The discovery that a variety of genes carried on plasmids are transposable strengthens the view that bacterial replicons are assembled on a modular principle. Transposons are therefore turning out to be valuable pointers to the past, present and future evolution of bacteria.

Richardson and Lanka seem to be mainly tetranucleotides with sequences ACCC and ACCA(C) respectively. No specific sequences seem to be present in the RNA primers isolated from 